\title{
Variations in the expression of TIMP1, TIMP2 and TIMP3 in cutaneous melanoma with regression and their possible function as prognostic predictors
}

\author{
SABINA ZURAC ${ }^{1,2}$, MONICA NEAGU $^{3}$, CAROLINA CONSTANTIN $^{3}$, MIRELA CIOPLEA $^{1,2}$, ROXANA NEDELCU $^{2}$, \\ ALEXANDRA BASTIAN $^{1,2}$, CRISTIANA POPP ${ }^{1}$, LUCIANA NICHITA ${ }^{1,2}$, RAZVAN ANDREI ${ }^{1}$, TIBERIU TEBEICA ${ }^{2}$, \\ CRISTIANA TANASE ${ }^{3}$, VIRGINIA CHITU ${ }^{1,2}$, CONSTANTIN CARUNTU ${ }^{2}$, MIHAELA GHITA $^{2}$, CATALIN POPESCU $^{1,2}$, \\ DANIEL BODA $^{2}$, BOGDAN MASTALIER ${ }^{1,2}$, NICOLETA MARU ${ }^{2}$, CLAUDIU DAHA $^{2}$, BOGDAN ANDREESCU ${ }^{1}$, \\ IOAN MARINESCU ${ }^{1}$, ADRIAN REBOSAPCA ${ }^{1}$, FLORICA STANICEANU ${ }^{1,2}$, GABRIELA NEGROIU ${ }^{4}$, \\ DANIELA A. ION ${ }^{2}$, DRAGANA NIKITOVIC ${ }^{5}$, GEORGE N. TZANAKAKIS ${ }^{5}$, \\ DEMETRIOS A. SPANDIDOS ${ }^{6}$ and ARISTIDIS M. TSATSAKIS ${ }^{7}$
}

${ }^{1}$ Department of Pathology, Colentina University Hospital, Bucharest 020125; ${ }^{2}$ Department of Physiology,

'Carol Davila' University of Medicine and Pharmacy, Bucharest 050474; ${ }^{3}$ Department of Immunology,

'Victor Babes' National Institute of Pathology, Bucharest 050096; ${ }^{4}$ Institute of Biochemistry of Romanian Academy, Bucharest 060031, Romania; ${ }^{5}$ Department of Anatomy-Histology-Embryology, Faculty of Medicine, University of Crete, Heraklion 71003; ${ }^{6}$ Laboratory of Clinical Virology, Medical School, University of Crete, Heraklion 71409; ${ }^{7}$ Department of Forensic Sciences and Toxicology, Medical School, University of Crete, Heraklion 71003, Greece

Received January 26, 2016; Accepted March 29, 2016

DOI: $10.3892 / 01.2016 .4391$

\begin{abstract}
Regression in melanoma is a frequent biological event of uncertain prognostic value as the lesion exhibits heterogeneous phenotypical features, both at the morphological and immunohistochemical level. In the present study, we examined the expression of tissue inhibitors of metalloproteinases (TIMP1, TIMP2 and TIMP3) in melanoma with regression. We specifically examined the expression levels of these TIMPs in regressed components (RC) and non-regressed components (NRC) of the tumor and compared their expression levels with those in non-regressed melanomas. We found that TIMP1 was overexpressed in the NRC of melanomas with partial regression (PR) compared with the NRC in melanomas with segmental regression $(\mathrm{SR})(\mathrm{P}=0.011)$. TIMP2 was overexpressed in the NRC of melanomas with PR compared with the NRC in melanomas with $\mathrm{SR}$ (PR/SR, $\mathrm{P}=0.009)$; or compared with the NRC in melanomas with simultaneous SR-PR $(\mathrm{P}=0.002)$; or compared with melanomas without regression (absence of regression) $(\mathrm{P}=0.037)$. Moreover, TIMP3 was overexpressed in the NRC of all melanomas with SR as
\end{abstract}

Correspondence to: Professor Monica Neagu, Department of Immunology, 'Victor Babes' National Institute of Pathology, 99-101 Splaiul Independentei, Bucharest 050096, Romania E-mail: neagu.monica@gmail.com

Key words: melanoma with regression, tissue inhibitors of metalloproteinases, prognosis compared to the $\mathrm{RC}$ component $(\mathrm{P}=0.007)$. Our findings on the differential expression of TIMP1, TIMP2 and TIMP3 in melanomas with regression support the hypothesis that the morphological differences identified in the melanoma regression spectrum may have a correlation with prognosis. This may explain the controversial findings within the literature concerning the biological and prognostic role of regression in melanoma.

\section{Introduction}

Cutaneous melanoma is one of the most aggressive human malignancies, with an increasing incidence worldwide. Currently, this incidence is high among young patients and is accompanied with an increased mortality rate, despite latest advances in therapy. The lack of adequate treatment strategies for melanoma is an important public health concern, and remains a challenge for the dermatologist, the pathologist and the oncologist. Of note, melanoma is one of the few tumors that can present spontaneous tumor regression due to complex interactions between the cancer cells and the host; this process occurs relatively frequently (10-35\% of cases), with an even higher incidence in thin melanomas $(60 \%$ of cases with a Breslow index of $<0.75 \mathrm{~mm}$ ) (1-6).

Tumor regression in melanoma involves the destruction of tumor cells by the host immune system, whereas its histopathological appearance is characterized by the infiltration of inflammatory cells, such as lymphocytes with melanophages, and by vascular hyperplasia and fibrosis. Indeed, several morphological types of regression may occur [partial regres- 
sion (PR), segmental regression (SR) and complete regression]. We have previously investigated the morphological characteristics of regression in melanoma and correlated these to the expression of matrix metalloproteinases (MMPs) (6).

The role of regression in the prognosis of melanoma has not yet been well-established, with some authors supporting a favorable prognosis, whereas others have argued that tumor regression in melanoma may be a marker for a poor prognosis (7-16). An unfavorable prognosis in melanoma with regression is supported by bibliographic cases of histopathologically verified complete tumor regression with the simultaneous presence of lymphatic and/or visceral metastases $(17,18)$. Among the patients at the Department of Pathology, Colentina University Hospital (Bucharest, Romania), we detected 3 additional cases of completely regressed melanomas whose identification was based on presenting metastases. These are very dramatic cases with a significant psychological impact both on the patients and their families, as well as on the medical staff. However, to the best of our knowledge, we are unaware of the existence of melanoma cases with complete regression without metastasis as a clinical presentation; nobody will biopsy cutaneous scars, even if there is a history of a previously spontaneously vanishing tumor in the same spot, if the person is otherwise healthy.

In view of the existing controversies on the prognostic significance of regression in melanoma, it may be helpful to assume that we can encounter both progression and regression in the same tumor. This process would be the result of complex interactions between tumor cells and the host immune system, with the final outcome depending on the efficacy of the defense mechanisms. It seems that the most important mechanism involved in the process of tumor regression is the action of cytotoxic T lymphocytes and natural killer cells $(19,20)$. Importantly, during the progression of this insidious disease, both the inflammatory and angiogenic response have been shown to correlate with the remodeling of the melanoma extracellular matrix (ECM) (21). Moreover, alterations in the melanoma microenvironment have been shown to correlate with melanoma vertical progression (22), as well as with the regulation of its key biological functions (23-25). Alterations in the ECM may be attributed to varying expression patterns between melanoma cells and melanocytes (26), or to the activity of proteolytic enzymes, including MMPs, which in addition to the remodeling of the ECM, also shed cell membrane receptors and release mediators, thus regulating ECM-melanoma cell interactions (27).

In view of the fact that the interactions between the tumor cells and the surrounding microenvironment play an important role in the evolution of cutaneous melanoma, in this study, we examined the expression of tissue inhibitors of metalloproteinases (TIMPs) in regressed melanoma.

\section{Materials and methods}

We selected 93 cases of superficial spreading melanoma (SSM) and nodular melanoma (NM) consecutively diagnosed between January 2007 and December 2008 at the Department of Pathology, Colentina University Hospital. All the specimens were received for histopathological diagnosis. Fragments of tumor and/or nontumoral tissue where harvested according to guidelines of medical practice in pathology (Ministry of Health Regulation no. 1217/September 16, 2010 published in Official Monitor no. 723/October 29, 2010, annex 1); tissue fragments were routinely processed and paraffin-embedded; 3 micron-thick sections were cut and routinely stained with hematoxilin and eosin (H\&E). Based on the microscopic examination of H\&E stained slides, the histopathologic diagnosis of melanoma was established and several histopathological characteristics were evaluated: Breslow index, the Clark level of invasion into adjacent normal structures, ulcerations, vascular invasion, perineural invasion, intratumoral inflammatory infiltrates, cellular pleomorphism, the mitotic index, intratumoral vascularity, satellite/in-transit metastasis, as well as lymphatic or visceral metastases when specific biopsies were performed.

This study was approved by the Ethics Committee of Colentina University Hospital, and written informed consent was obtained from all patients for the use of the human specimes for experimental purposes.

For the purpose of this study, additional sections were prepared and immunohistochemical analyses for TIMP1, TIMP2 and TIMP3 were duly performed. The specific details of the primary antibodies used are listed in Table I. The detection system used was Novolink Polymer (Leica/Novocastra, Leica Biosystems, Wetzlar, Germany) with DAB as a chromogen. A semiquantitative score to record the level of staining was utilized as follows: absent (-), faint positivity (+), moderate positivity (++) and intense positivity (+++).

We separated our cases into 2 groups, one of melanoma with regression, and the other of melanoma without regression [absence of regression (AR)]. We further stratified the cases with regression based on the respective morphologic type as follows: i) SR, complete regression of tumor cells with the simultaneous preservation of the proliferating cells in other parts of the melanoma; ii) PR, partial disappearance of tumor cells with partial replacement by inflammatory cells, melanophages and fibrosis; and iii) SR-PR, the simultaneous presence of SR and RP areas, observed in a few cases (7).

In total, we analyzed 3 categories of tumor tissue segments: for the subgroup of the melanoma with regression, we analyzed areas with regression [regressed component (RC) of the melanoma with regression], as well as areas without regression [non-regressed component (NRC) of the melanoma with regression], whereas one type of tumor tissue of the AR subgroup was examined. The level of expression recorded for each marker was compared between the NRC versus the RC in the same tumor, as well as in the NRC versus AR among different tumors. For the better appreciation of the differences between the NRC and RC, components we subtracted the immunohistochemical score for the RC from that of the NRC (i.e., $\mathrm{NRC}+++$ and $\mathrm{RC}++$ : $\mathrm{NRC} / \mathrm{R}=+1$; $\mathrm{NRC}++$ and $\mathrm{RC}+++$ : $\mathrm{NRC} / \mathrm{RC}=-1)$.

Statistical analysis was performed using the EXCEL and EPIINFO programs; a P-value of $<0.05$ was considered to indicate a statistically significant difference (calculated by the $\chi^{2}$ test).

\section{Results}

In total, we analyzed a group of 93 melanoma specimens including 62 SSMs (66.66\%) and 31 NMs (33.33\%). The 
Table I. Primary antibodies used in this study.

\begin{tabular}{|c|c|c|c|c|c|c|c|}
\hline No. & Primary antibody & Clone & Catalogue no. & Host & Source & Pre-treatment ${ }^{\mathrm{a}}$ & Dilution \\
\hline 1 & TIMP1 & 6F6a & NCL-TIMP1-485 & Mouse & Leica, Wetzlar, Germany & HIER, EDTA citrate, $\mathrm{pH} 8$ & $1: 300$ \\
\hline 2 & TIMP2 & $46 \mathrm{E} 5$ & NCL-TIMP2-487 & Mouse & Leica, Wetzlar, Germany & HIER, EDTA citrate, $\mathrm{pH} 8$ & $5: 240$ \\
\hline 3 & TIMP3 & $18 \mathrm{D} 12 \mathrm{~b}$ & NCL-TIMP3 & Mouse & Leica, Wetzlar, Germany & HIER, EDTA citrate, $\mathrm{pH} 8$ & $0.5: 1,000$ \\
\hline
\end{tabular}

${ }^{\text {a}} \mathrm{HIER}$, heat induced epitope retrieval; TIMP, tissue inhibitor of metalloproteinase.

A
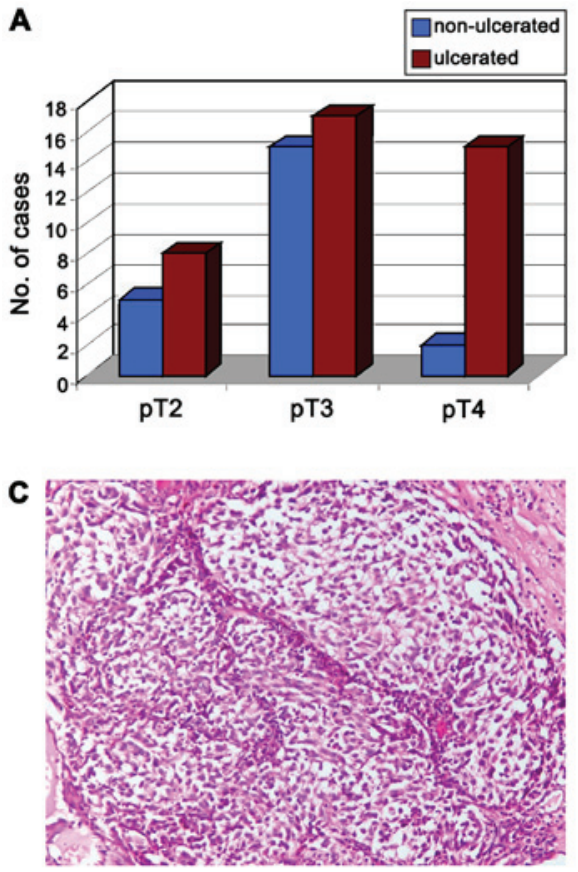

B

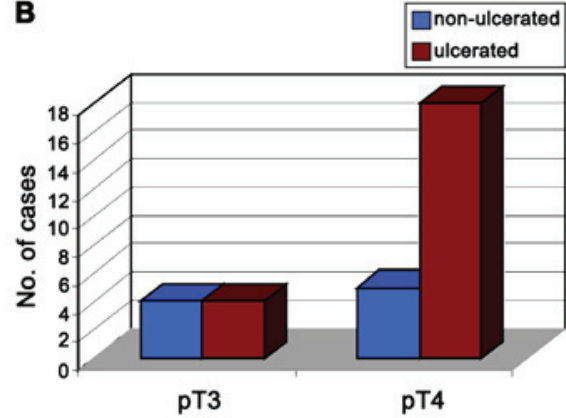

D

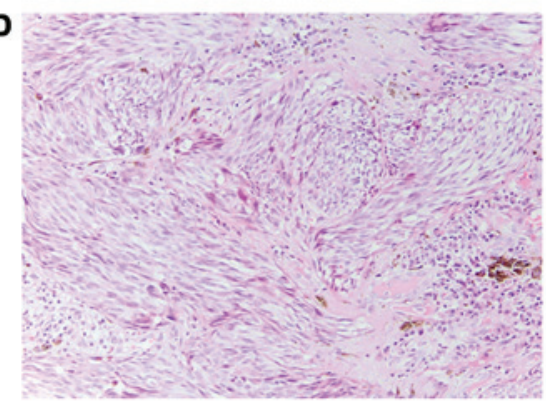

Figure 1. Histopathological aspects of melanomas. (A) Distribution of superficial spreading melanomas according to the pT stage. (B) Distribution of nodular melanomas according to the pT stage. (C) Nests of epithelioid cells in non-regressed part of superficial spreading melanoma with regression. Hematoxylin and eosin (H\&E) staining, x200 magnification. (D) Nests and fascicles of spindle cells in nodular melanoma. H\&E, x200 magnification.

majority of the tumors were at the pT4 stage $(86.02 \%)$ and/or ulcerated $(66.67 \%)$. In both tumor types, the majority of cases were at the pT3 and pT4 stage $(79.03 \%$ of SSMs and all cases of NMs) (Fig. 1).

Regression was present in the SSM cases; 39 SSMs $(62.90 \%)$ presented regression: 13 cases $(33.33 \%)$ of SR, 17 cases $(43.58 \%)$ of PR and 9 cases $(23.07 \%)$ with areas of SR-PR in different parts of the tumor.

TIMP expression in the NRC component compared with the AR cases. The TIMP1, TIMP2 and TIMP3 expression levels were similar in both the NRC and AR cases. There was obvious TIMP1 overexpression in PR cases $[76.47 \%$ cases with PR were intensely positive $(+++)$ for TIMP1 compared to $23.07 \%$ of $\mathrm{SR}$ cases that had the same feature $(\mathrm{PR} / \mathrm{SR}, \mathrm{P}=0.011)$; Figs. 2A-C and 3A]. In additoin, the PR cases exhibited intense positivity for TIMP2 as compared to cases with other types of regression or $\mathrm{AR}$ cases (PR/SR, $\mathrm{P}=0.009$; $\mathrm{PR} / \mathrm{SR}-\mathrm{PR}, \mathrm{P}=0.002$; PR/AR, P=0.037; Figs. 2D-F and 3B]. No differences were noted in TIMP3 expression among the different types of regression (Fig. 2G-I).
TIMPS expression in regressed and non-regressed areas in the same tumor. All TIMPs had a similar pattern of expression in melanoma with regression: some cases exhibited a loss of expression of TIMPs in the NRC versus the RC (TIMP1 and TIMP2, $7.69 \%$ of cases; TIMP3, $12.82 \%$ of cases), some presented with the overexpression of TIMPs in the NRC versus the RC (TIMP1, 66,66\%; TIMP2, 61.53\%; TIMP3, 64.10\%), whereas almost a quarter of the cases had similar TIMP expression (TIMP1, 25.41\%; TIMP2, 30.76\%; TIMP3, 23.07\%) (Fig. 3C and E). There were no differences observed between the NRC and RC in TIMP1 or TIMP2 expression according to the type of regression; TIMP3 was overexpressed in all SR cases when comparing the NRC to the RC component $(\mathrm{P}=0.007$; Fig. 3F).

\section{Discussion}

ECM remodeling has been shown to closely correlate with the progression of melanoma $(22,27)$. Numerous biomolecules are involved in this process with TIMPs being one of the key regulators. The functions of TIMPs are complex as 

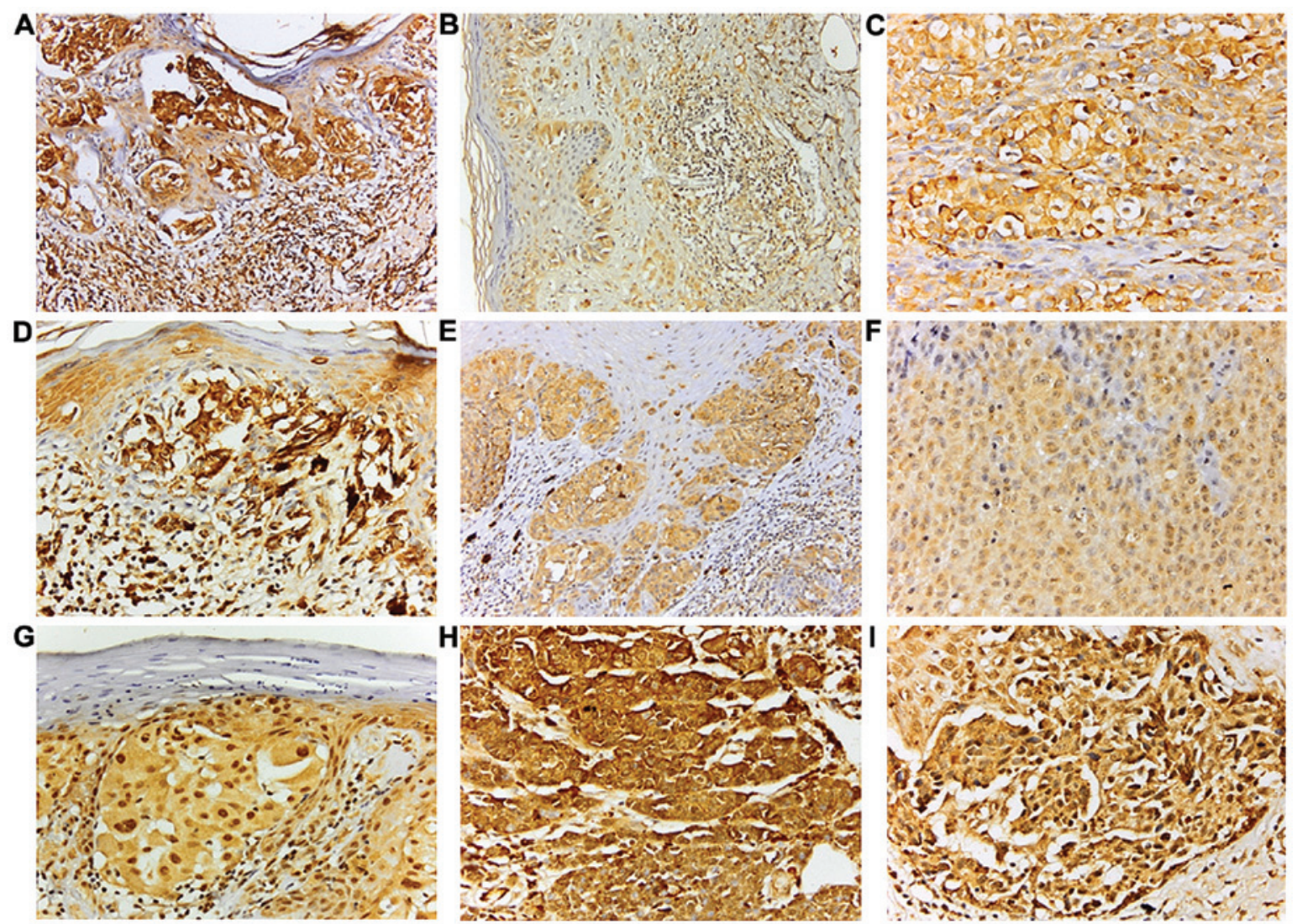

Figure 2. Expression of tissue inhibitors of metalloproteinases (TIMPs) in regressed and non-regressed melanoma. (A) Intense diffuse expression of TIMP1 in areas of partial regression (x200 magnification); (B) Faint diffuse positivity of TIMP1 in areas of partial and segmental regression (x200 magnification), (C) Faint diffuse positivity for TIMP1 in melanomas without regression (x400 magnification). (D) Intense diffuse expression of TIMP2 in areas of partial regression (x400 magnification); (E) Faint diffuse positivity of TIMP2 in areas of partial and segmental regression (x200 magnification). (F) Faint diffuse positivity for TIMP2 in melanomas without regression (x400 magnification). (G-I) Similar expression of TIMP3 in (G) areas of partial regression, x400 magnification, (H) non-regressed component of melanoma with regression, x400 magnification, or (I) melanomas without regression, x200 magnification.

A

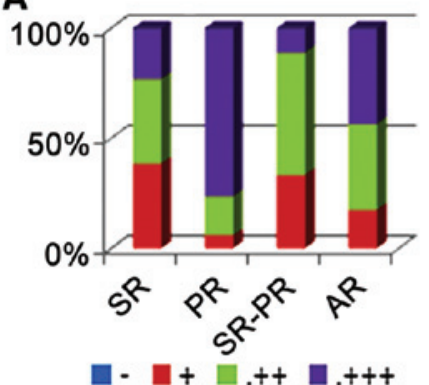

D

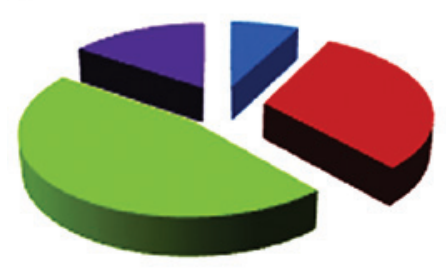

$\square .-1 \square 0 \quad+1 \square .+2$
B

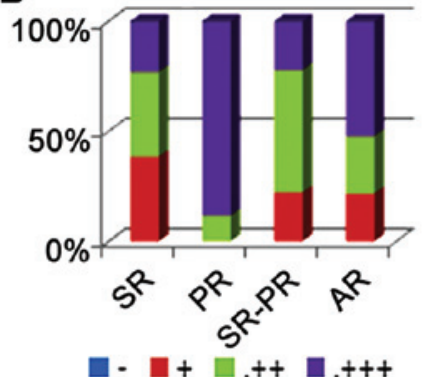

$\mathbf{E}$

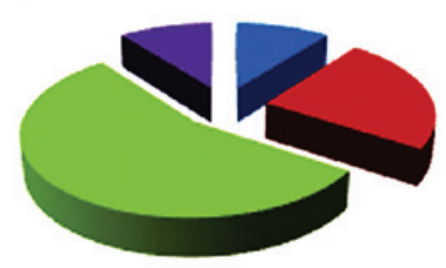

$\square .-1 \square 0 \quad \square .1 \square .+2$
C

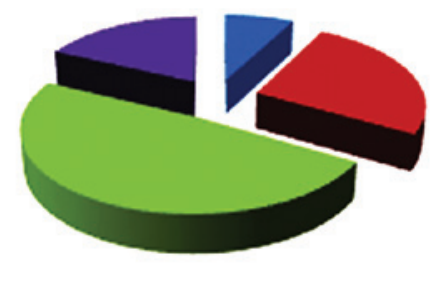

$\square .-1 \backsim 0 \square .+1 \square .+2$

$\mathbf{F}$

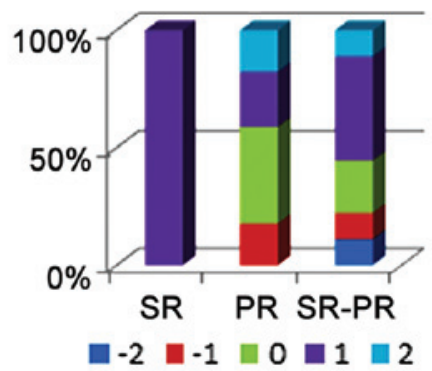

Figure 3. Expression of tissue inhibitors of metalloproteinases (TIMPs) in different types of regression. (A) TIMP1 expression in non-regressed component (NRC) and absence of regression (AR) according to the type of regression. (B) TIMP2 expression in NRC and AR according to the type of regression. (C) Differences in TIMP1 expression in NRC versus RC in melanoma. (D) Differences in TIMP2 expression in NRC versus RC in melanoma. (E) Differences in TIMP3 expression in NRC versus RC in melanoma. (F) Differences in TIMP3 expression in NRC versus RC based on type of regression. 
they participate in the progression of melanoma at several levels. Thus, TIMPs participate in the regulation of MMP activity (28-30). This function is perpetrated by both the $\mathrm{N}$-terminal and C-terminal domain of TIMPs; the N-terminal domain binds to the active site of MMPs, thus preventing the access of substrates to the catalytic site of MMP, while the C-terminal domain binds to the hemopexin-like domain of pro-MMP-9 and pro-MMP-2 (31). TIMPs are involved in the cell adhesion process and in the subsequent modulation of cell growth through several mechanisms, including the regulation of cytoskeletal organization, the direct interaction with cell adhesion molecules or regulating the expression of specific ECM components (32).

TIMPs exhibit anti-angiogenic activities by inhibiting MMP-dependent angiogenesis. TIMP3 affects angiogenesis through the direct regulation of vascular endothelial growth factor receptor-2 (VEGFR2) activity and the inhibition of VEGF-A mitogenic effects, whereas the effects of TIMP2 are more complex, as it inhibits both endothelial cell growth and the migration of these cells $(33,34)$. The latter is accomplished through TIMP 2 interaction with $\alpha 3 \beta$ receptor or by enhancing the reversion-inducing-cystein-rich protein with Kazal motif (RECK) expression, which inhibits several downstream biomolecules, such as MMP-2, MMP-9, MT1-MMP, ADAM10, the final effect being the loss of cell migration $(33,34)$. TIMPs have also been reported to modulate apoptosis with different outcomes, either exhibiting pro-apoptotic activity [TIMP3 through the stabilization of tumor necrosis factor receptor 1 (TNFR-I) and Fas] or anti-apoptotic activity (TIMP1 and TIMP2) $(33,34)$. Importantly, TIMP expression has been shown to be associated with other pathological and toxicological conditions $(35,36)$.

We have previously investigated MMP-1, MMP-2, MMP-3, MMP-9, MMP-11 and MMP-13 expression in melanomas with and without regression (6). Indeed, the differential expression of the MMPs, MMP-1 and MMP-11, in non-regressed tumors as compared to melanoma without regression, with a possible improvement in prognosis, for the former group of cases, was reported (6).

In the present study, we examined TIMP1, TIMP2 and TIMP3 expression in regressed versus non-regressed melanoma. We failed to identify differences in the expression of the 3 TIMPs (TIMP1, TIMP2 and TIMP3) between the NRC and AR or between the RC and NRC. However, interesting results were obtained when particular forms of regression were analyzed. Our examination of the morphological features of melanoma regression suggest that the destruction of tumor cells in melanoma regression may present different histological phenotypes, indicating a different spectrum of alteration, with SR seeming to bestow a more favorable potential (6).

In the present study, TIMP3 was overexpressed in all SR cases in the NRC versus the RC tumor segments. Of note, a recent study on metastatic lymph node melanoma demonstrated an inverse negative correlation of TIMP 3 expression with intratumor vessel density (37). Das et al (37) thus concluded that 'TIMP3 gene silencing by promoter methylation is associated with a poor outcome'. Moreover, TIMP3 gene promoter methylation has been shown to positively correlate with melanoma brain metastases, incurring a poor prognosis (poor disease-free survival and overall survival) (38).
Our present findings however, demonstrate that PR cases overexpressed TIMP2 in the NRC more often than AR or in the NRC of melanomas with other types of regression. A recently published study, in line with the above, demonstrated that the overexpression of TIMP2 suppressed the proliferation of melanoma cell lines by inhibiting the activation of the Wnt/ $\beta$-catenin pathway (39). In this study, TIMP1 was overexpressed in the NRC of PR cases as compared to AR cases. This finding is difficult to evaluate, since the role of TIMP1 in carcinogenesis is unclear. Since TIMP1 negatively regulates the activity of several MMPs involved in ECM degradation, it is likely that it inhibits tumor development and progression. In some tumors, TIMP1 expression correlates with a less aggressive tumor behavior and, conversely, factors that downregulate TIMP1 are associated with an unfavorable prognosis (poor overall survival and short disease-free interval) $(32,40)$.

However, TIMP1 may also promote tumor progression by intervening in several key cellular processes, including apoptosis (anti-apoptotic activity) and anoikis resistance $(41,42)$ Specifically, the activation of the PI3K pathway resulting in the assembly of a supramolecular complex containing TIMP1, CD63 and $\beta 1$-integrins, has been suggested (43). Several studies have correlated TIMP1 overexpression in melanoma with an unfavorable prognosis (31-33): some authors suggest that increased serum TIMP1 levels correlate with a poor prognosis in patients with unresectable stage melanoma (43). Moreover, concomitant high serum levels of TNFR-II, transforming growth factor (TGF)- $\alpha$, TIMP1 and C-reactive protein (CRP) are associated with a poor outcome in melanoma (44). Indeed, tumors overexpressing TIMP1 progress more rapidly and have an increased tendency towards metastases (45).

Particular types of regression may occur due to different immunological mechanisms. Our previous studies on inflammatory infiltrates in melanoma with regression suggest that Langerhans cells are constantly associated with thinner tumors (46), whereas the presence of nodular infiltrates of Langerhans cells in areas of regression are statistically associated with the presence of Langerhans cells in the main tumor mass (47-49). Indeed, particular types of regression (from an immunological point of view) may bestow a more favorable prognosis than other forms of regression or AR.

In conclusion, further progress in understanding cancer biology, as regards tumor cell proliferation, tumor microenvironment and host response is in order $(29,50,51)$. However, differences in the biological behavior of tumors sharing the same origin indicate, without equivoque, that the mechanisms involved in tumorigenesis and progression are not yet completely deciphered. Further studies are warranted, both to further analyze the tumor omics, as well as to identify novel tumor biomarkers for diagnosis and potential therapeutic targets.

In this study, we described the differences in the expression of TIMP1, TIMP2 and TIMP3 in regressed and non-regressed areas of melanoma with regression. TIMP3 was overexpressed in all SR cases in the NRC as compared to the RC component. Moreover, a tendency towards TIMP1 and TIMP2 overexpression in the NRC in melanomas with PR as compared to AR cases was evident. These findings support the hypothesis that the morphological differences identified in the melanoma regression spectrum may correlate with prognosis, thus explaining 
the controversial findings within the literature concerning the biological and prognostic role of regression.

\section{Acknowledgements}

The authors would like to thank the physicians from the Colentina Hospital in Bucharest and the Dermato-Oncology Excellence Centre in Bucharest for providing access to patients diagnosed with cutaneous melanoma, as well as for their clinical monitoring. This study was financially supported by the Sectorial Operational Programme Human Resources Development, financed from the European Social Fund and by the Romanian Government under the contract nos. POSDRU/159/1.5/S/137390 and POSDRU/89/1.5/S/60746, 'Carol Davila' University of Medicine and Pharmacy, Bucharest for Young Researchers grant no. 33891/2014 and the Executive Agency for Higher Education, Research, Development and Innovation (UEFISCDI) under the contract no. PN-II-PT-PCCA-2013-4-1407 (project no. 190).

\section{References}

1. Alquier-Bouffard A, Franck F, Joubert-Zakeyh J, Barthélémy I, Mansard S, Ughetto S, Aublet-Cuvelier B, Déchelotte PJ, Mondié JM, Souteyrand P and D'incan M: Regression in primary cutaneous melanoma is not predictive for sentinel lymph node micrometastasis. Ann Dermatol Venereol 134: 521-525, 2007 (In French).

2. McGovern VJ, Shaw HM and Milton GW: Prognosis in patients with thin malignant melanoma: Influence of regression. Histopathology 7: 673-680, 1983.

3. Abramova L, Slingluff CL Jr and Patterson JW: Problems in the interpretation of apparent 'radial growth phase' malignant melanomas that metastasize. J Cutan Pathol 29: 407-414, 2002.

4. Balch CM, Gershenwald JE, Soong SJ, Thompson JF, Atkins MB, Byrd DR, Buzaid AC, Cochran AJ, Coit DG, Ding S, et al: Final version of 2009 AJCC melanoma staging and classification. J Clin Oncol 27: 6199-6206, 2009.

5. Zurac S, Negroiu G, Petrescu S, Andrei R, Tebeica T, Popp C, Musţată R, Neagu M, Constantin C, Solovan C, et al: Spectrum of morphologic alterations of regression in cutaneous melanoma - potential for improving disease prognosis. Rom $\mathrm{J}$ Intern Med 50: 145-153, 2012.

6. Andrei R, Zurac S, Socoliuc C and Staniceanu F: Variation in expression of metalloproteinases in cutaneous melanoma with regression, possible indicator of tumor heterogeneity. DermatoVenerol (Buc) 60: 133-145, 2015.

7. Trau H, Kopf AW, Rigel DS, Levine J, Rogers G, Levenstein M, Bart RS, Mintzis MM and Friedman RJ: Regression in malignant melanoma. J Am Acad Dermatol 8: 363-368, 1983.

8. Kaur C, Thomas RJ, Desai N, Green MA, Lovell D, Powell BW and Cook MG: The correlation of regression in primary melanoma with sentinel lymph node status. J Clin Pathol 61: 297-300, 2008

9. Shaw HM, Rivers JK, McCarthy SW and McCarthy WH: Cutaneous melanomas exhibiting unusual biologic behavior. World J Surg 16: 196-202, 1992.

10. Guitart J,Lowe L, Piepkorn M, Prieto VG, Rabkin MS, Ronan SG, Shea CR, Tron VA, White W and Barnhill RL: Histological characteristics of metastasizing thin melanomas: A case-control study of 43 cases. Arch Dermatol 138: 603-608, 2002.

11. Blessing $\mathrm{K}$ and McLaren KM: Histological regression in primary cutaneous melanoma: Recognition, prevalence and significance. Histopathology 20: 315-322, 1992.

12. Paladugu RR and Yonemoto RH: Biologic behavior of thin malignant melanomas with regressive changes. Arch Surg 118: 41-44, 1983.

13. Oláh J, Gyulai R, Korom I, Varga E and Dobozy A: Tumour regression predicts higher risk of sentinel node involvement in thin cutaneous melanomas. Br J Dermatol 149: 662-663, 2003.
14. Socrier Y, Lauwers-Cances V, Lamant L, Garrido I, Lauwers F, Lopez R, Rochaix P, Chevreau C, Payoux P, Viraben R, et al: Histological regression in primary melanoma: Not a predictor of sentinel lymph node metastasis in a cohort of 397 patients. Br J Dermatol 162: 830-834, 2010

15. Fontaine D, Parkhill W, Greer W and Walsh N: Partial regression of primary cutaneous melanoma: Is there an association with sub-clinical sentinel lymph node metastasis? Am J Dermatopathol 25: 371-376, 2003.

16. Liszkay G, Orosz Z, Péley G, Csuka O, Plótár V, Sinkovics I, Bánfalvi T, Fejõs Z, Gilde K and Kásler M: Relationship between sentinel lymph node status and regression of primary malignant melanoma. Melanoma Res 15: 509-513, 2005.

17. Emanuel PO, Mannion M and Phelps RG: Complete regression of primary malignant melanoma. Am J Dermatopathol 30: 178-181, 2008.

18. High WA, Stewart D, Wilbers CRH, Cockerell CJ, Hoang MP and Fitzpatrick JE: Completely regressed primary cutaneous malignant melanoma with nodal and/or visceral metastases: A report of 5 cases and assessment of the literature and diagnostic criteria. J Am Acad Dermatol 53: 89-100, 2005.

19. Klebanoff CA, Acquavella N, Yu Z and Restifo NP: Therapeutic cancer vaccines: Are we there yet? Immunol Rev 239: 27-44, 2011.

20. Faries MB and Morton DL: Therapeutic vaccines for melanoma: Current status. BioDrugs 19: 247-260, 2005.

21. van Kempen LC, van Muijen GN and Ruiter DJ: Stromal responses in human primary melanoma of the skin. Front Biosci 10: 2922-2931, 2005

22. Nikitovic D, Mytilinaiou M, Berdiaki A, Karamanos NK and Tzanakakis GN: Heparan sulfate proteoglycans and heparin regulate melanoma cell functions. Biochim Biophys Acta 1840: 2471-2481, 2014

23. Chalkiadaki G, Nikitovic D, Berdiaki A, Katonis $P$, Karamanos NK and Tzanakakis GN: Heparin plays a key regulatory role via a p53/FAK-dependent signaling in melanoma cell adhesion and migration. IUBMB Life 63: 109-119, 2011.

24. Chalkiadaki G, Nikitovic D, Katonis P, Berdiaki A, Tsatsakis A, Kotsikogianni I, Karamanos NK, and Tzanakakis GN: Low molecular weight heparin inhibits melanoma cell adhesion and migration through a $\mathrm{PKCa} / \mathrm{JNK}$ signaling pathway inducing actin cytoskeleton changes. Cancer Lett 312: 235-244, 2011.

25. Nikitovic D, Assouti M, Sifaki M, Katonis P, Krasagakis K, Karamanos NK and Tzanakakis GN: Chondroitin sulfate and heparan sulfate-containing proteoglycans are both partners and targets of basic fibroblast growth factor-mediated proliferation in human metastatic melanoma cell lines. Int J Biochem Cell Biol 40: 72-83, 2008.

26. Sifaki M, Assouti M, Nikitovic D, Krasagakis K, Karamanos NK and Tzanakakis GN: Lumican, a small leucine-rich proteoglycan substituted with keratan sulfate chains is expressed and secreted by human melanoma cells and not normal melanocytes. IUBMB Life 58: 606-610, 2006.

27. Moro N, Mauch C and Zigrino P: Metalloproteinases in melanoma. Eur J Cell Biol 93: 23-29, 2014.

28. Kalluri R and Weinberg RA: The basics of epithelial-mesenchymal transition. J Clin Invest 119: 1420-1428, 2009.

29. Hanahan D and Weinberg RA: Hallmarks of cancer: The next generation. Cell 144: 646-674, 2011.

30. Slootweg PJ and Zurac S: Prognostic and predictive value of epithelial to mesenchymal transition-associated markers in oral squamous cell carcinoma. Curr Proteomics 10: 218-227, 2013.

31. Duan JX, Rapti M, Tsigkou A and Lee MH: Expanding the activity of tissue inhibitors of metalloproteinase (TIMP)-1 against surface-anchored metalloproteinases by the replacement of its C-terminal domain: Implications for anti-cancer effects. PLoS One 10: e0136384, 2015.

32. Bourboulia D and Stetler-Stevenson WG: Matrix metalloproteinases (MMPs) and tissue inhibitors of metalloproteinases (TIMPs): Positive and negative regulators in tumor cell adhesion. Semin Cancer Biol 20: 161-168, 2010.

33. Baker AH, Edwards DR and Murphy G: Metalloproteinase inhibitors: Biological actions and therapeutic opportunities. J Cell Sci 115: 3719-3727, 2002.

34. Visse R and Nagase H: Matrix metalloproteinases and tissue inhibitors of metalloproteinases: Structure, function, and biochemistry. Circ Res 92: 827-839, 2003.

35. Kovatsi L, Batzios S, Nikolaou K, Fragou D, Njau S, Tsatsakis A, Karakiulakis G and Papakonstantinou E: Alterations in serum MMP and TIMP concentrations following chronic heroin abuse. Toxicol Mech Methods 23: 377-381, 2013. 
36. Tsarouhas K, Soufla G, Apostolakis S, Zaravinos A Panagiotou M, Khoury M, Hassoulas JA, Tsatsakis AM and Spandidos DA: Transcriptional regulation of TIMPs in ascending aorta aneurysms. Thromb Res 126: 399-405, 2010.

37. Das AM, Koljenović S, Oude Ophuis CM, van der Klok T, Galjart B, Nigg AL, van Cappellen WA, Noordhoek Hegt V, Dinjens WN, Atmodimedjo PN, et al: Association of TIMP3 expression with vessel density, macrophage infiltration and prognosis in human malignant melanoma. Eur J Cancer 53: $135-143,2016$.

38. Gonzalez-Gomez P, Bello MJ, Alonso ME, Amiñoso C, Lopez-Marin I, De Campos JM, Isla A, Gutierrez M and Rey JA: Promoter methylation status of multiple genes in brain metastases of solid tumors. Int J Mol Med 13: 93-98, 2004.

39. Xia Y and Wu S: Tissue inhibitor of metalloproteinase 2 inhibits activation of the $\beta$-catenin signaling in melanoma cells. Cell Cycle 14: 1666-1674, 2015.

40. Wen X, Wu JQ, Peng W, Feng JF and Tang JH: MicroRNA-377 predicts poor clinical outcome of gastric cancer and induces tumorigenesis by targeting multiple tumor-suppressor genes. Oncol Rep 34: 203-210, 2015.

41. Toricelli M, Melo FH, Peres GB, Silva DC and Jasiulionis MG: Timp1 interacts with beta-1 integrin and CD63 along melanoma genesis and confers anoikis resistance by activating PI3-K signaling pathway independently of Akt phosphorylation. Mol Cancer 12: 22, 2013

42. Chunhacha P, Sriuranpong V and Chanvorachote P: Epithelial-mesenchymal transition mediates anoikis resistance and enhances invasion in pleural effusion-derived human lung cancer cells. Oncol Lett 5: 1043-1047, 2013.

43. Kluger HM, Hoyt K, Bacchiocchi A, Mayer T, Kirsch J, Kluger Y, Sznol M, Ariyan S, Molinaro A and Halaban R: Plasma markers for identifying patients with metastatic melanoma. Clin Cancer Res 17: 2417-2425, 2011.
44. Tarhini AA, Lin Y, Yeku O, LaFramboise WA, Ashraf M, Sander C, Lee S and Kirkwood JM: A four-marker signature of TNF-RII, TGF- $\alpha$, TIMP-1 and CRP is prognostic of worse survival in high-risk surgically resected melanoma. J Transl Med 12: 19, 2014.

45. Ricca TI, Liang G, Suenaga AP, Han SW, Jones PA and Jasiulionis MG: Tissue inhibitor of metalloproteinase 1 expression associated with gene demethylation confers anoikis resistance in early phases of melanocyte malignant transformation. Transl Oncol 2: 329-340, 2009.

46. Zurac S, Negroiu G, Andrei R, Petrescu S, Tebeica T, Petre M, Neagu M,Constantin C, Chitu V, Salavastru C, et al: Inflammatory infiltrate in melanoma with regression as prognostic parameter. Virchows Arch 463: 127, 2013.

47. Neagu M, Constantin C and Zurac S: Immune parameters in the prognosis and therapy monitoring of cutaneous melanoma patients: Experience, role, and limitations. BioMed Res Int 2013: 107940, 2013.

48. Neagu M: The immune system - a hidden treasure for biomarker discovery in cutaneous melanoma. Adv Clin Chem 58: 89-140, 2012.

49. Bulman A, Neagu M and Constantin C: Immunomics in skin cancer - improvement in diagnosis, prognosis and therapy monitoring. Curr Proteomics 10: 202-217, 2013.

50. Spandidos DA: A unified theory for the development of cancer. Biosci Rep 6: 691-708, 1986.

51. Spandidos DA: The cancer story. Cancer Biol Ther 3: 1184-1186, 2004. 